\title{
ANÁLISIS DE LAS PROPIEDADES MECÁNICAS EN BLOQUES DE CEMENTO CON INCORPORACIÓN DE PLÁSTICO, REFORZADO CON POLIESTIRENO REVESTIDO EN MALLA HEXAGONAL GALVANIZADA
}

\section{ANALYSIS OF THE MECHANICAL PROPERTIES IN CEMENT BLOCKS WITH INCORPORATION OF PLASTIC, EMPHASIZED TONE WITH POLYSTYRENE COATED IN HEXAGONAL GALVANIZED MESH}

\author{
MSc. Haidee Yulady Jaramillo*, MSc. Fernando Jesús Regino** \\ MSc. July Andrea Gómez** \\ * Universidad Francisco de Paula Santander Ocaña, Facultad de Ingenierías, Grupo de \\ Investigación en Construcción Geotecnia y Medio Ambiente GIGMA. \\ Ocaña, Norte de Santander, Colombia. \\ E-mail: hyjaramillo@ufpso.edu.co. \\ ** Universidad Francisco de Paula Santander Ocaña, Facultad de Ingenierías, Grupo de \\ Investigación en Ingenierías Aplicadas para la Innovación Gestión y Desarrollo INGAP \\ Ocaña, Norte de Santander, Colombia. \\ E-mail: (fjreginou, jagomezc) @ufpso.edu.co.
}

\begin{abstract}
Resumen: En el presente artículo se exponen los resultados de un trabajo investigativo de diseño experimental, estos obtenidos a partir de ensayos de compresión a un bloque de cemento con adición de plástico triturado y poliestireno revestido en malla hexagonal galvanizada con una relación de cemento: agua: plástico de 1:1:1,5.
\end{abstract}

Palabras clave: Plástico, poliestireno, bloque, malla hexagonal galvanizada.

\begin{abstract}
In the present article the results of a investigating designing experimental job, these once essays of compression were gotten from to a cement block with addition of crushed plastic and polystyrene coated in hexagonal mesh galvanized with a relation of cement expose themselves: Water: Plastic of 1:1:1.5.
\end{abstract}

Keywords: Plastic, polystyrene, block, hexagonal galvanized mesh

\section{INTRODUCCIÓN}

La mayoría de los materiales plásticos que se fabrican, especialmente los destinados a la industria alimentaria para envases y embalajes, tienen como característica fundamental la resistencia al ataque microbiano lo que permite definirlos como no biodegradables. Por este motivo, si bien no son contaminantes directos, dificultan su recolección y disposición final, produciendo un impacto ambiental que conlleva a múltiples trastornos ecológicos (Magariños, et al., 1998). Cada día desechamos grandes cantidades de polímeros en forma de botellas de plástico, cartones y envases de yogurt. Los expertos calculan que el $25 \%$ de los residuos poliméricos no puede reciclarse, por tres motivos principales: contiene mezclas de tipos diferentes de polímeros; no es rentable económicamente; y es un proceso demasiado sucio (Flores et al., 2014).

El plástico, una de las innovaciones más significativas del siglo XX, y es un material común en todos lados. En los últimos años se observa un crecimiento sustancial en el consumo de plástico en todo el mundo, lo que también aumenta la 
producción de residuos relacionados con el plástico. Los desechos plásticos son ahora un verdadero problema ambiental, están hechos a partir de varios químicos tóxicos, y por lo tanto, el plástico contamina el suelo, el aire y el agua (Saikia y Brito, 2012). Y ya que el plástico es un material no biodegradable, su aprovechamiento en bloques para construcción significaría un aprovechamiento y manejo de dicho material.

La amenaza de los residuos plásticos parece estar aumentando cada vez más. Muchos países han restringido el uso de bolsas de plástico y muchos están en proceso de hacerlo. El relleno de plástico también es peligroso debido a su lenta tasa de degradación y su naturaleza voluminosa. La masa residual puede dificultar el flujo de agua subterránea y también puede bloquear el movimiento de las raíces. Los desechos plásticos también contienen varios elementos tóxicos, especialmente cadmio y plomo, que pueden mezclarse con agua de lluvia y contaminar el suelo y el agua (Saikia y Brito, 2012).

Por otra parte el poliestireno expandido o poliestireno EPS es un material plástico, derivado del poliestireno, que se utiliza sobre todo en el sector de la construcción y del envasado. Químicamente hablando, el poliestireno es un polímero termoplástico que se obtiene de la polimerización del estireno monómero.

Es un perfecto aislante térmico, en el ámbito de la construcción, este plástico se utiliza para revestir fachadas por ser un gran aislante térmico del calor y del frío y a la hora de reciclar poliestireno EPS es posible y muy beneficioso para el medio ambiente. Una de las máximas cualidades de este producto es que solo el $2 \%$ de su composición es plástico y el $98 \%$ restante, es aire.

Esto lo convierte en un material que, de primeras, no contamina directamente ni el aire ni el agua. Pero aun así, tarda unos 1.000 años en degradarse por lo que su acumulación sí podría resultar perjudicial para el entorno.(Gutierrez, 2014); (R García et al., 2018).

Se ha observado un crecimiento sustancial en el consumo de plástico en todo el mundo en los últimos años, lo que ha llevado a enormes cantidades de desechos relacionados con el plástico. La Reutilización también puede contribuir a reducir el impacto ambiental producto de los desechos plásticos en el medio ambiente. (Culligan, 2013), (Martinez, 2019), así mismo el reciclaje de residuos plásticos para producir nuevos materiales como hormigón o mortero parece ser una de las mejores soluciones para la eliminación de residuos plásticos, debido a sus ventajas económicas y ecológicas. Se han realizado o se están realizando varios trabajos para evaluar las propiedades de los compuestos de cemento que contienen varios tipos de desechos plásticos como agregado, relleno o fibra. Con la presente investigación se proponen la elaboración de elementos constructivos como los ladrillos a partir de cemento, plástico e icopor, como parte de estrategias de reciclaje que aprovechen estos residuos como agregados en mortero.

\section{Antecedentes}

El reciclaje es una estrategia fundamental frente al creciente volumen de residuos, es por ello que cada vez más son los proyectos e investigaciones que buscan alternativas para solucionar esta problemática.

El sector de la construcción es uno de los principales responsables de la generación de residuos, contaminación, trasformación del entorno y uso considerable de energía. Se estima que cerca del $40 \%$ de las materias primas en el mundo, que equivalen a 3000 millones de toneladas por año, son destinadas para la construcción. así sucede con el $17 \%$ del agua potable (WorldGBC, 2008), el $10 \%$ de la tierra (UNEP- SBCI, 2006) y el $25 \%$ de la madera cultivada, valor que asciende a $70 \%$ si se considera el total de recursos madereros (Edwards, 2001). El sector constructor es también el responsable de más de un tercio del consumo de energía en el mundo, en su mayoría durante el tiempo de habitación y uso del inmueble. Un $20 \%$ de la energía es consumida durante el proceso de construcción, elaboración de materiales y demolición de las obras de construcción (UNEPSBCI, 2009); (J Plaza, M Núñez, 2017).

Adicionalmente la generación de residuos sólidos y de agentes contaminantes también es un problema ambiental asociado al sector de la construcción, el cual es el principal generador de los gases de efecto invernadero en muchos países (Agudelo et al., 2012). Estas emisiones alcanzaban 8.6 billones de toneladas métricas en el 2004, según el Panel Intergubernamental sobre el Cambio Climático, cifra que podría alcanzar los 15.6 billones de toneladas métricas para el 2030, en caso de continuar construyendo de la manera actual (UNEP, 2009). Sólo en el caso del CO2 , las 
edificaciones producen el $33 \%$ de las emisiones de este gas (WorldGBC, 2008), que son, en la actualidad, motivo de numerosos proyectos de investigación. Asimismo, la construcción es responsable de la generación del $30 \%$ de los residuos sólidos mundiales, demostrando la necesidad de generar métodos y aplicar prácticas que reduzcan la cantidad de residuos en vista del agotamiento del espacio para su adecuada disposición (UNEP-SBCI, 2006). Esta problemática se traduce en costos a causa de la degradación ambiental que, para Colombia, supera el 3.7\% del PIB por año (Sánchez et al., 2007), por el aumento en la frecuencia de desastres naturales y la degradación de los suelos por la modificación del entorno, y el deterioro de la salud por contaminación del aire y del agua, en las zonas urbanas, donde ya habita más del $50 \%$ de la población mundial (UNFPA, 2007).

Con lo base en lo anterior, es necesario que la industria de la construcción tome partida en los esfuerzos que se hacen mundialmente para contrarrestar el cambio climático, y este proyecto está enfocado a contribuir en dichos esfuerzos, planteando una alternativa para el aprovechamiento de los residuos plásticos que se generan en la ciudad de Ocaña.

\section{Materiales y Métodos}

\subsection{Caracterización de los materiales}

\subsubsection{Plástico triturado}

El plástico es un material que tiene grandes propiedades, pero cuando hablamos de su reciclaje destaca una por encima de otras: tiene mucho volumen y poca densidad, esto es, ocupa mucho y pesa poco.

El proceso de trituración nos proporciona un material homogéneo, del mismo tamaño y una forma muy similar, independientemente de la forma y del tamaño que tengan originariamente.

Se pueden depositar botellas de plástico, sillas, cajas, que resultará en un material pequeño y, sobre todo, fácil de transportar y de almacenar. (Recytrans, 2015).

\subsubsection{Poliestireno expandido}

El poliestireno en espuma puede tener más de 95 por ciento de aire y se usa como aislante doméstico y de electrodomésticos, envase protector liviano, tablas para surf, servicio de alimentos y envasado de alimentos, repuestos automotrices, sistemas de estabilización de caminos y acotamientos y mucho más. Además, es valorado por sus propiedades de aislamiento y acolchado. (Chemicalsafetyfacts.org, 2015)

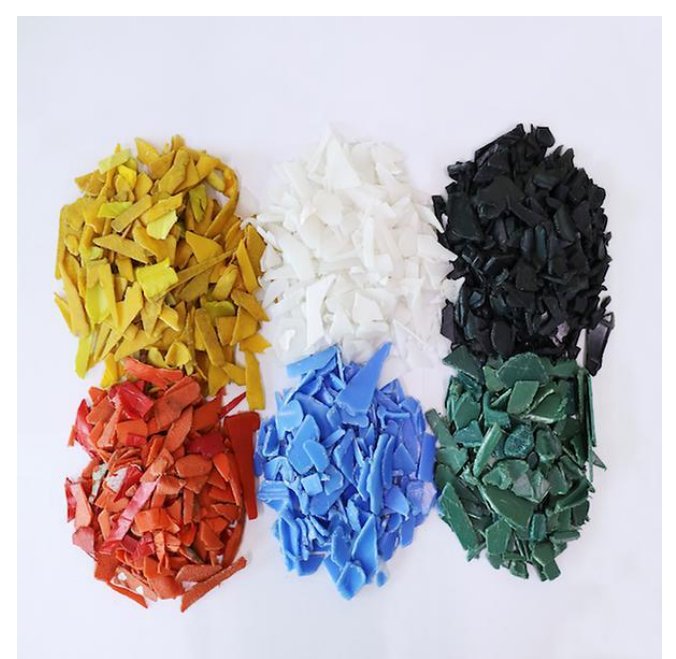

Fig. 1. Plástico triturado.

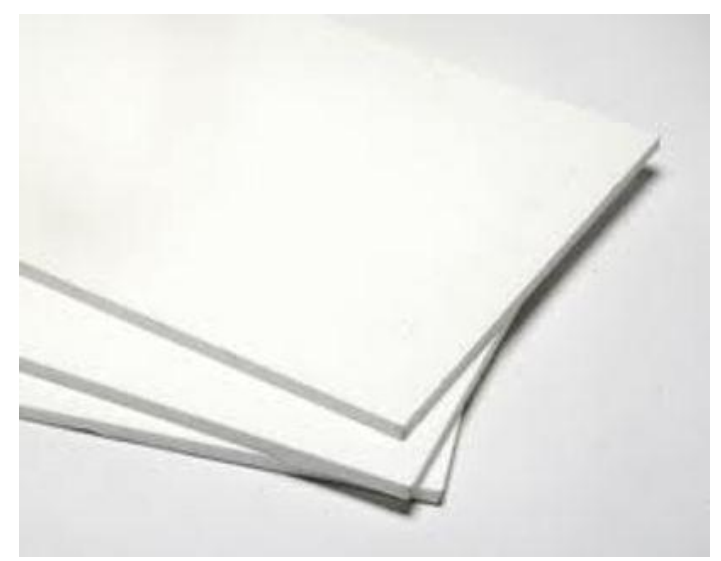

Fig. 2. Poliestireno Expandido.

\subsubsection{Cemento}

El cemento empleado en la realización de los ensayos fue el comercialmente distribuido por la empresa ARGOS, es cemento tipo 1 o de uso general, y su ficha técnica se resume en la Tabla I.

Tabla 1: Parámetros químicos y físicos del cemento

\begin{tabular}{cccc}
\hline Parámetros & Especificaci & NTC 321 & ASTM C- \\
químicos & ones & Tipo I & 1157 tipo \\
& ARGOS & & GU. \\
\hline
\end{tabular}




\begin{tabular}{|c|c|c|c|}
\hline $\begin{array}{l}\text { Óxido de } \\
\text { magnesio, } \\
\text { Mgo, Max } \\
(\%)\end{array}$ & 6.00 & 7.00 & - \\
\hline $\begin{array}{l}\text { Trióxido de } \\
\text { azufre, } \mathrm{so}_{3}, \\
\text { Max }(\%)\end{array}$ & 3.5 & 3.5 & - \\
\hline $\begin{array}{l}\text { Parámetros } \\
\text { físicos }\end{array}$ & $\begin{array}{c}\text { Especificaci } \\
\text { ones } \\
\text { ARGOS }\end{array}$ & $\begin{array}{c}\text { NTC } 121 \\
\text { Tipo I }\end{array}$ & $\begin{array}{c}\text { ASTM C- } \\
1157 \text { tipo } \\
\text { GU. }\end{array}$ \\
\hline $\begin{array}{l}\text { Fraguado } \\
\text { inicial }^{(1)} \text {, } \\
\text { mínimo } \\
\text { (minutos) }\end{array}$ & 45 & 45 & 45 \\
\hline $\begin{array}{l}\text { Fraguado } \\
\text { inicial }^{(1)} \text {, } \\
\text { mínimo } \\
\text { (minutos) }\end{array}$ & 420 & 480 & 420 \\
\hline $\begin{array}{l}\text { Expansión } \\
\text { autoclave, } \\
\text { Max }(\%)\end{array}$ & 0.8 & 0.8 & 0.8 \\
\hline $\begin{array}{l}\text { Expansión en } \\
\text { agua }^{(2)} \text {, Max } \\
(\%)\end{array}$ & 0.02 & - & 0.02 \\
\hline $\begin{array}{l}\text { Resistencia a } \\
3 \text { días }^{(3)} \text {, Min } \\
\text { (Mpa) }\end{array}$ & 9.0 & 8.0 & 13.0 \\
\hline $\begin{array}{l}\text { Resistencia a } \\
7 \text { días }^{(3)}, \text { Min } \\
\text { (Mpa) }\end{array}$ & 16.0 & 15.0 & 20.0 \\
\hline $\begin{array}{l}\text { Resistencia a } \\
28 \text { días }^{(3)} \text {, Min } \\
\text { (Mpa) }\end{array}$ & 26.0 & 24.0 & 28.0 \\
\hline $\begin{array}{l}\text { Blaine, } \\
\text { Mínimo } \\
\left(\mathrm{cm}^{3} / \mathrm{gr}\right)\end{array}$ & 2800 & 2800 & - \\
\hline
\end{tabular}

\subsubsection{Malla hexagonal}

Es una malla de alambre galvanizado tejido con aberturas hexagonales enlazada en triple torsión, fortalecida con remates laterales, que es comúnmente usada para galpones avícolas, jaulas para diferentes especies, construcción para el control de fisuras en morteros (concreto) domésticos, entre otras cosas.

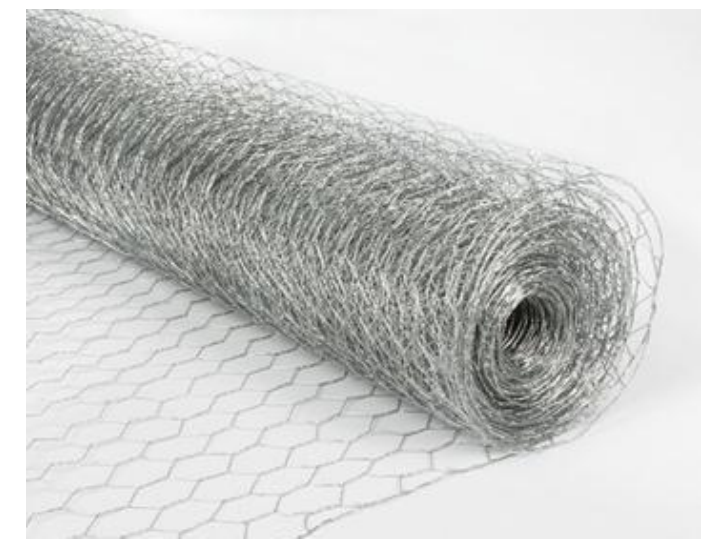

Fig. 3. Malla hexagonal galvanizada.

\subsection{Diseño experimental}

La metodología planteada se basó en experiencias realizadas con plástico PET (tereftalato de polietileno) en diversas partes del mundo, el refuerzo que compone el ladrillo fue invención propia a partir de observaciones realizadas sobre su comportamiento en elementos estructurales y arquitectónicos de los dos materiales usados, el poliestireno y malla galvanizada respectivamente. Dicho esto se plantearon las siguientes fases:

Fase 1: Caracterización de los materiales.

Fase 2: Obtención de los materiales necesarios para el bloque.

Fase 3: Construcción de la formaleta para el bloque.

Fase 4: Corte del poliestireno expandido en las medidas necesarias para ser empleado en el bloque. Fase 5: Enmallado del poliestireno.

Fase 6: Medición del agua, plástico y cemento necesario para la mezcla.

Fase 7: Engrasado del molde para el bloque.

Fase 8: Armado del bloque.

Fase 9: Tiempo de fraguado (24 horas).

Fase 10: Curado de 5 días.

Fase 11: Ensayo de compresión.

Fase 12: Análisis de resultados.

Para la obtención de los materiales necesarios para el bloque en cuestión, se hizo un sondeo por las diversas zonas de Ocaña para saber dónde se podían obtener los materiales más económicos y el plástico triturado nos fue suministrado por una docente de la universidad francisco de paula Santander Ocaña. Así mismo, se fue a una carpintería para la construcción de la formaleta del bloque, una vez se tuvieron todos los elementos necesarios para la construcción de este, y un vez adquiridos todos los insumos necesarios se procedió al desarrollo de la investigación. 
La malla utilizada para este proyecto cumple con una resistencia de $45 \%$ más que un alambre suave normal y es tres veces más resistente a la corrosión que las convencionales, el poliestireno, más conocido como icopor se usó ya que posee gran capacidad de aislamiento térmico y acústico y además posee baja densidad lo que le permite ocupar grandes volúmenes conllevando así a un ahorro en cemento y cualquier aditivo que se haya utilizado, es un material limpio, versátil y fácil de manejar. (Casas, 2016), (Acero BSV, Venta de acero, distribución y comercialización de productos derivados del acero, 2017).

\section{RESULTADOS}

Inicialmente se realizó la búsqueda del material plástico adecuado, tanto por su origen como por el tamaño de partícula el cual se recomienda debe estar por debajo de los $3 \mathrm{~mm}$, ya que con una mayor superficie especifica que potenciara la adición entre el plástico y el cemento que cumple la función de aglutinante.

Luego se procedió a enmallar el icopor con malla galvanizada, este tiene unas medidas de $10.5 \mathrm{~cm}$ por $23.5 \mathrm{~cm}$ y con un ancho de $1 \mathrm{~cm}$ buscando principalmente que se cubra la matriz del ladrillo.

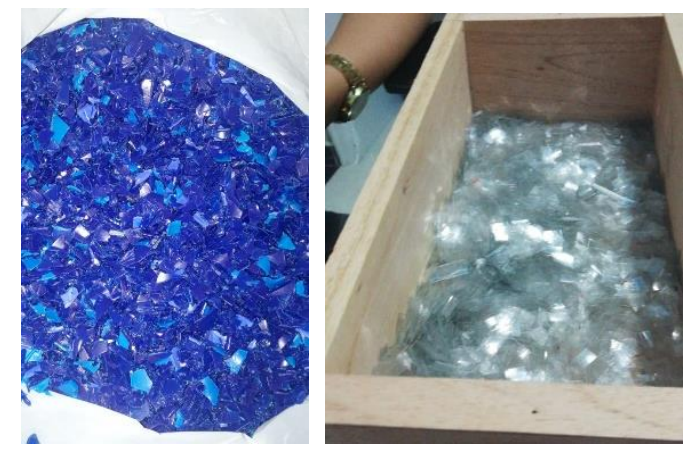

Fig. 4. Plástico triturado.

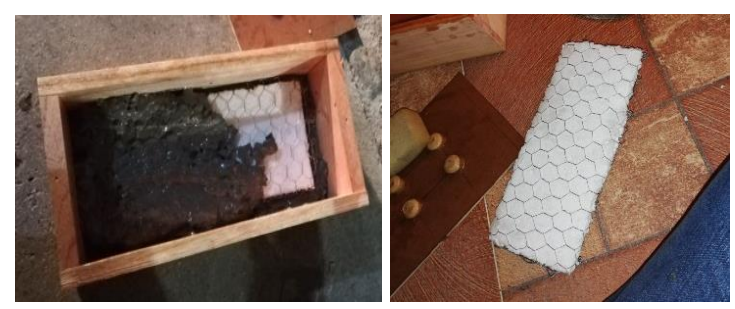

Fig. 5. Icopor enmallado

El ladrillo consta de dos rectángulos de icopor cada uno ubicado a $2.5 \mathrm{~cm}$ desde el inicio del cilindro que de alto mide $8 \mathrm{~cm}$.

Universidad de Pamplona I. I. D. T. A.
Posteriormente se realizó la dosificación para el diseño de mezclas, que se estableció en una proporción 1:1.5:1 correspondiente a una parte de cemento por 1,5 partes de plástico y una parte de agua.

Los pesos utilizados para la elaboración de un bloque se detallan en la Tabla 2.

\section{Tabla 2: Cantidades para cada ladrillo}

\begin{tabular}{cc}
\hline Material & Cantidad (Gr) C/u \\
\hline Cemento & 1540 \\
Plástico & 1365 \\
Agua & 1260 \\
\hline Total & 4165 \\
\hline
\end{tabular}

Las probetas fueron realizadas con unas medidas de $26.5 \mathrm{~cm}$ de ancho x $12.5 \mathrm{~cm}$ de largo x $8.5 \mathrm{~cm}$ de alto respectivamente, luego de la mezcla de cemento, plástico y agua como se muestra en la figura 6 se procedió a vaciar la pasta en una formaleta con las dimensiones antes mencionadas.
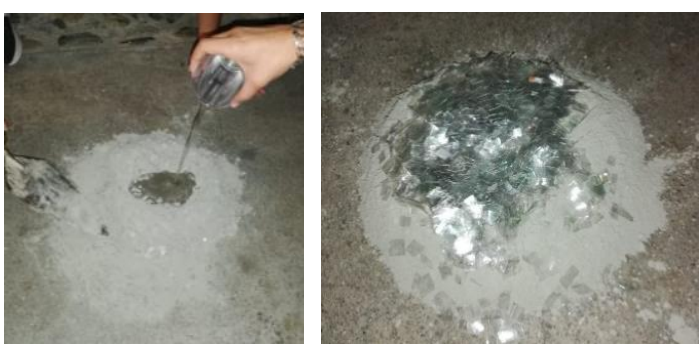

Fig. 6. Mezcla de plástico cemento y agua

Finalmente se procede a dejar fraguar la mezcla por 24 horas posteriormente se hizo el respectivo curado de la probeta en agua con cal a partir de las especificaciones establecidas en las norma NTC 1377, como se muestra en la figura 7.

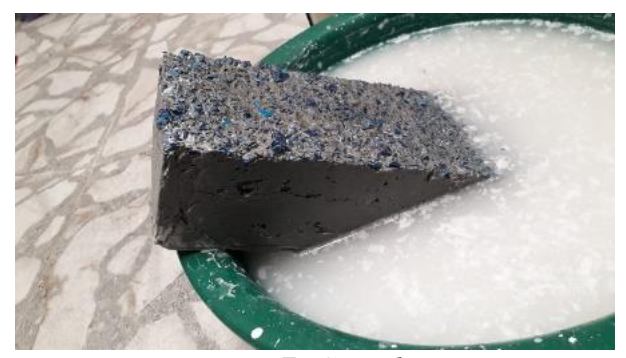

Fig. 7. Curado

\subsection{Ensayos de resistencia}

El ensayo de compresión simple fue realizado con una maquina universal y los resultados obtenidos se muestran en la tabla 3 . 
Tabla 3: Resistencia a la compresión a los 7 días

\begin{tabular}{cc}
\hline Probeta & Resistencia (MPa) \\
\hline 1 & 2,105 \\
2 & 2,201 \\
3 & 2,039 \\
\hline Prom & 2,115 \\
\hline
\end{tabular}

En la figura 8 se puede apreciar una de las probetas posterior al ensayo de compresion.

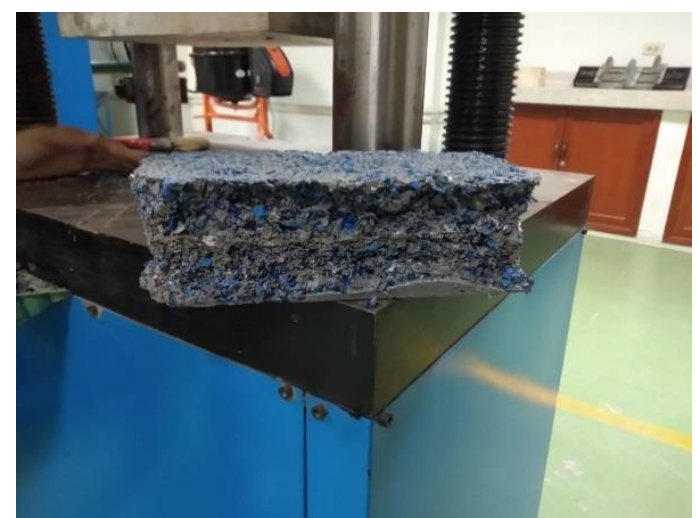

Fig. 8. Probeta después del ensayo de compresión

Es interesante mencionar que el tipo de falla no corresponde a las que generalmente se pueden apreciar en elementos de concreto, ya que no hubo una fractura del material, ni tampoco superficies de falla claramente definidas.

\section{CONCLUSIONES}

El sector de la construcción es uno de los principales dinamizadores de la economía mundial y constituye una necesidad para el progreso y el desarrollo de la sociedad, esto implica que aun cuando sea uno de los principales responsables de la contaminación y cambio climático no puede ser frenado, por lo cual se debe recurrir a mecanismos de innovación para la redacción de los residuos y el aprovechamiento de los mismo, principalmente los relacionados a los plásticos.

Con base en los resultados obtenidos mediante los ensayos de resistencia se pude concluir inicialmente que los bloques representan una opción como elementos de mampostería, mas no como elementos estructurales, puesto que las resistencias alcanzadas a los 7 días no son suficientes para elementos que deban transmitir cargas. Sin embargo, es necesario el desarrollo de ensayos adicionales, y con mayores tiempos de curado para poder definir exactamente la resistencia del bloque.
Con respecto al tipo de falla que presenta el bloque, es interesante que no se ajusta a las fallas convencionales que presentan los elementos en concreto, en los cuales hay fracturas y claros planos de falla, en este caso hubo un aplastamiento de la probeta, y se aprecia una considerable desintegración de la matriz que borde las partículas plásticas, esto podría corresponder a que no hay una adecuada adhesión entre el cemento y el plástico, en dicho caso sería necesario reducir el tamaño de las partículas del plástico para aumentar la superficie especifica.

Finalmente, el bloque propuesto es un primer modelo de elemento constructivo, que principalmente logra reducir hasta un $40 \%$ la densidad de un bloque estándar de concreto con las mismas dimensiones, y con base en los valores alcanzados es necesario replantear las dosificaciones para mejorar las resistencias, aunque como ya se planteó, el modelo propuesto funciona como elemento divisorio.

\section{REFERENCIAS}

Acero BSV. (2017) Venta de acero, distribución y comercialización de productos derivados del acero. $\quad$ https://acerobsv.com/mallahexagonal.html (Consultado el 28 de Julio de 2019)

Agudelo, H., Hernández, A. y Cardona, D. (2012). "Sostenibilidad: Actualidad y necesidad en el sector de la construcción en Colombia". Gestión y ambiente. Vol.15, No.1, p.105-117.

Casas, J. (2016) ICOFORMAS. CONSTRUCCION: https://www.icoformas.com/antigua/construcc ion.html (Consultado el 28 de Julio de 2019)

Chemicalsafetyfacts.org. (2015) Poliestireno https://www.chemicalsafetyfacts.org/es/polies tireno/ (Consultado el 28 de Julio de 2019)

Culligan. (2013) El impacto ambiental de los plásticos https://www.culligan.es/blog/elimpacto-ambiental-de-los-plasticos (Consultado el 28 de Julio de 2019)

Edwards, B. (2001). Guía básica de la sostenibilidad. Editorial Gustavo Gili S.A.

Gutiérrez, M. (2014). Ecología e Industria: Todas las novedades - Por qué reciclar poliestireno EPS:

http://blogs.uab.cat/ecologiaindustrial/2016/10 /21/por-que-reciclar-poliestireno-eps/ (Consultado el 28 de Julio de 2019) 
JEG Plaza, MAR Núñez, (2017) Formación en competencias específicas para la industria del software colombiano. Experiencias del uso del aprendizaje basado en proyectos. Revista Colombiana de Tecnologías de Avanzada, ISSN: 1692-7257.

Jaramillo, H. Y. (2016). Análisis de las características mecánicas en mezclas de mortero con la incorporación de fibras de hojas de maíz. 17th LACCEI International Multi-Conference for Engineering, Education, and Technology: "Industry, Innovation, And Infrastructure for Sustainable Cities and Communities", 3.

Magarinos, O. E., Alderete, C. E., Arias, L. E., \& Lucca, M. E. (1998). "Estudio de morteros que contienen escamas de plástico procedente de residuos post-industriales Study of mortars with industrial residual plastic scales". Mater. Constr, Vol. 250, p.33-52.

Martínez, I. (2019). Travesia. El problema del plástico en el medio ambiente https://travesiapirenaica.com/problemaplastico-en-el-medio-ambiente/ (Consultado el 01 de Agosto de 2019)

Recytrans. (15 de 05 de 2015). Recytrans. Obtenido de Trituración de plástico: https://www.recytrans.com/blog/trituracionde-plastico/ (Consultado el 01 de Agosto de 2019)

Saikia, N. De Brito, J. (2012). "Use of plastic waste as aggregate in cement mortar and concrete preparation: A review". Construction and Building Materials, Vol.34, p.385-401.

Sánchez E., Kulsum Ahmed, T., Awe, Y., Banco Mundial. (2007). Prioridades ambientales para la reducción de la pobreza en Colombia. Mayol Ediciones S.A.

UNEP- SBCI, (2006) Sustainable Buildings and Construction Initiative. http://www.unepsbci.org/SBCI_2006.pdf (Consultado el 02 de Agosto de 2009)

UNEP- SBCI. (2009). Sustainable Buildings and Climate Initiative, Greenhouse gas emission baselines and reduction potentials from buildings in South Africa: A discussion document.

http://www.unep.org/sbci/pdfs/SBCISAreport. pdf (Consultado el 02 de Agosto de 2009)
United Nations Population Fundation, UNFPA, (2007). Estado de la población mundial 2007. Indicadores demográficos, sociales y económicos

http://www.unfpa.org/swp/2007/spanish/notes /indicators/s_indicator2.pdf (Consultado el 02 de Agosto de 2019)

R García-León, E Florez, C Acevedo (2018). Caracterización térmica de mezclas de arcillas utilizadas en la fabricación de productos de mampostería para la construcción. Revista Colombiana de Tecnologías de Avanzada, ISSN: 1692-7257.

V. Flores, J. Rojas, R. Torres, R. Vallejos, P. Flores y M. Flores (2014). "Mezclas de cemento y agregados de plástico para la construcción de viviendas ecológicas". Revista Ciencias Tecnologías y Agrarias Vol. 16, p.101-110.

World Green Building Council. WorldGBC, (2008). Construction and WorldGBC to Collect Global Green Trends Data to Advance the Sharing of Green Information and Intelligence. McGrawHill. New York. 